\title{
A Esperança Matemática e Algumas das suas Áplicações à Teoria da Ámostragem
}

\author{
FREDERICO PIMENTEL GOMES
}

Professor Substituto de Matemática da E. S. A. "Luiz de Queiroz"

INDICE

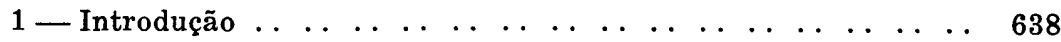

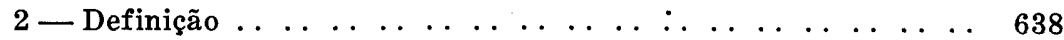

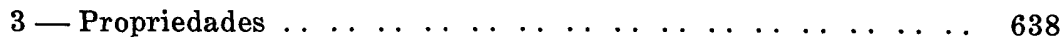

4 - Significado estatístico $\ldots \ldots \ldots \ldots . \ldots \ldots$

5 - Aplicações à teoria da amostragem . . . . . . . . . . . . . . 640

6 - Variável casual contínua . . . . . . . . . . . . . . . . . . 6464

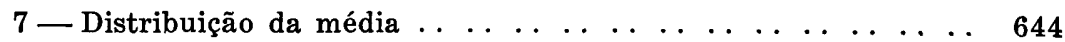

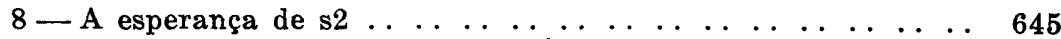

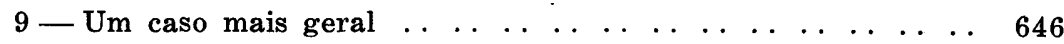

10 — Bibliografia citada . . . . . . . . . . . . . . . . . . . 64648 


\section{1. - INTRODUÇÃO}

Este trabalho consta de duas partes bem distintas. Na primeira delas, que abrange os capítulos $2,3,4$ e 5, examinamos o caso de uma variável casual definida num conjunto numerável. As demonstrações aí feitas são de carater elementar. $\mathrm{Na}$ segunda parte, em que discutimos o caso de uma variável contínua, somos forçados a lançar mão de métodos mais elevados, que estão fora do alcance dos que não têm conhecimentos especializados de Matemática.

De todos os métodos usados no estudo das distribuições das pequenas amostras o método das funções características nos parece o mais simples e elegante. Daí o fato de o termos preferido para as demonstrações que serão vistas. Os interessados acharão, porém, numa obra de KENDAL $\left[1,1^{\circ}\right.$ vol., pp. 231-253] ou num livro de USPENSKY [2, pp. 331-346], entre muitos, outros métodos interessantes que permitem resolver problemas análogos.

\section{2 - DEFINIÇÃO}

Se $x$ é uma variável casual ou aleatória, a esperança matemática de $x$, ou o seu valor médio é

$$
\mathrm{E}(\mathrm{x})=\mathrm{x} 1 \mathrm{p} 2+\mathrm{x} 2 \mathrm{p} 2+\ldots+\mathrm{xn} \mathrm{pn} .
$$

Supõe-se aí, implìcitamente, que o conjunto $\mathrm{C}$ em que está definida a variável $x$ é numerável.

\section{3 - PROPRIEDADES} temos

Antes de tudo, é evidente que, sendo $k$ uma constante,

$$
\underline{E}(\mathbf{k x})=\mathbf{k E}(\mathbf{x}) \text {. }
$$

Com efeito, podemos escrever:

$$
\begin{aligned}
E(k x) & =k x_{1} p_{1}+k x_{2} p_{2}+\ldots+k x_{n} p_{n} \\
& =k\left(x_{1} p_{1}+x_{2} p_{2}+\ldots+x_{n} p_{n}\right) \\
& =k E(x) .
\end{aligned}
$$

Consideremos, agora, duas variáveis casuais $x$ e $z \cdot$ Re

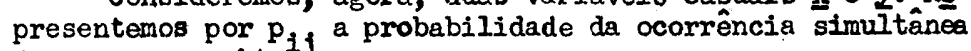
de $x_{i}$ e $y_{j}$ e obtembs:

$$
\begin{gathered}
E(x+y)=p_{11}\left(x_{1}+y_{1}\right)+p_{12}\left(x_{1}+y_{2}\right)+\ldots+p_{1 n}\left(x_{1}+y_{n}\right)+ \\
+p_{21}\left(x_{2}+y_{1}\right)+p_{22}\left(x_{2}+y_{2}\right)+\ldots+p_{2 n}\left(x_{2}+y_{n}\right)+
\end{gathered}
$$




$$
\begin{aligned}
& +p_{n 1}\left(x_{n}+y_{1}\right)+p_{n 2}\left(x_{n}+y_{2}\right)+\ldots+p_{n n}\left(x_{n}+y_{n}\right) . \\
& E(x+z)=x_{1}\left(p_{11}+p_{12}+\ldots+p_{1 n}\right)+x_{2}\left(p_{21}+p_{22}+\ldots+p_{2 n}\right)+ \\
& +\ldots+x_{n}\left(p_{n 1}+p_{n 2}+\ldots+p_{n n}\right)+z_{1}\left(p_{11}+p_{21}+\ldots+p_{n 1}\right)+ \\
& +z_{2}\left(p_{12}+p_{22}+\ldots+p_{n 2}\right)+\ldots+y_{n}\left(p_{1 n}+p_{2 n}+\cdots+p_{n n}\right) . \\
& P_{1}=p_{11}+p_{12}+\ldots+p_{\text {in }} \text {. } \\
& q_{1}=p_{11}+p_{21}+\ldots+p_{n i} \text {. }
\end{aligned}
$$

Logo obtemos

$$
\begin{aligned}
E(x+y) & =x_{1} P_{1}+x_{2} P_{2}+\ldots+x_{n} P_{n}+ \\
& +y_{1} Q_{1}+x_{2} Q_{2}+\ldots+x_{n} Q_{n}, \\
& \therefore E(x+y)=E(x)+E(y) .
\end{aligned}
$$

Suponhamos, agore, que $\mathrm{x}$ ○ I sejam variáveis casuais independentes, isto $\dot{\theta}$, que a probabilidade $p_{1 j}$ de ocorrerem simulténecmente $x_{1} \oplus J_{j}$ seja

$$
p_{1 j}=P_{1} \cdot Q_{j},
$$

onde $P_{1} \dot{e}$ a probabilidade de ocorrer $x_{1}, \oplus Q_{j}$, a de ocor-
rer $J_{j}$ Temos, então,

$$
\begin{aligned}
& E(x y)=p_{11} x_{1} y_{1}+p_{12} x_{1} y_{2}+\ldots+p_{1 n} x_{1} y_{n}+ \\
& +p_{21} x_{2} y_{1}+p_{22} x_{2} y_{2}+\ldots+p_{2 n} x_{2} y_{n}+ \\
& \text { + [.............................. } \\
& +p_{n 1} x_{n} y_{1}+p_{n 2} x_{n} y_{2}+\ldots+p_{n n} x_{n} y_{n} \text {, } \\
& E(x y)=\left(P_{1} x_{1}+P_{2} x_{2}+\ldots+P_{n} x_{n}\right)\left(Q_{1} y_{1}+Q_{2} y_{2}+\cdots+Q_{n} y_{n}\right) \\
& =E(x) \cdot E(y)
\end{aligned}
$$

Se k $\dot{e}$ uma constante, temos ainda

$$
\begin{aligned}
E(k) & =k p_{1}+k p_{2}+\ldots+k p_{n} \\
& =k\left(p_{1}+p_{2}+\ldots+p_{n}\right) \\
& =k .
\end{aligned}
$$




\section{4 - SIGNIFICADO ESTATISTICO}

Suponhamos que o conjunto de valores da variável casual $x$ constitui uma população de $N$ elementos. A probabilidade de extrair um determinado $x$ qualquer será $1 / \mathrm{N}$. Logo teremos

$$
E(x)=\frac{1}{N} x_{1}+\frac{1}{N} x_{2}+\ldots+\frac{1}{N} x_{N}=\frac{\Sigma_{x}}{N}=z
$$

Logo $E(x)$ è a média aritmética $\bar{x}$ da população.

Temos ainde

$$
\begin{aligned}
& E(x-\bar{x})=E(x)-E(\bar{x})=\bar{x}-\bar{x}=0 \\
& E\left[(x-\bar{x})^{2}\right]=E\left(x^{2}-25 x+\frac{2}{x}\right) \\
& =E\left(x^{2}\right)-2 \bar{x} E(x)+E\left(x^{2}\right) \\
& =\mathrm{E}\left(x^{2}\right)-2 x^{2}+x^{2} \\
& =\mathrm{E}\left(x^{2}\right)-\frac{2}{x}^{2} \\
& =\frac{\Sigma x^{2}}{N}-\left[\frac{\Sigma x}{1}\right]^{2} \\
& =\frac{\Sigma x^{2}-\left(\frac{\Sigma x}{N}\right)^{2}}{N}=\sigma^{2}=\frac{\Sigma(x-\bar{x})^{2}}{N} \text {. }
\end{aligned}
$$

Logo $E(x-\bar{x})^{2}$ não $\dot{e}$ mais que a variância $\sigma^{2}$.

\section{5 - APLICAÇÕES A TEORIA DA AMOSTRAGEM}

Suponhamos que da população constituída de todos os valores de $x$ extraímos, ao acaso, uma amostra de $n$ elementos. Podemos, então, com os dados da amostra, tentar estimar os parâmetros $\tilde{\mathrm{x}} \mathrm{e} \sigma 2 \mathrm{da}$ população. Somos tentados a tomar

$$
x=\frac{\Sigma x}{n} \text { e } s^{2}=\frac{\Sigma(x-\bar{x})^{2}}{n}
$$


como estimativas, respectivamente, de $\tilde{\mathrm{x}}$ e de $\sigma 2$. A conveniência das estimativas, de um modo geral, é um problema complexo, que não abordaremos agora. Uma exigência usual, porém, é que a estimativa deve ser "unbiased", isto é, imparcial ou não tendenciosa. Diz-se que uma estimativa $p$ de um parâmetro P da população é "unbiased" quando temos

$$
E(p)=P \text {. }
$$

Será que as estimativas $\bar{Z}$ e $s^{2}$ são imparcials?

Para $\bar{x}$ temos logo

$$
E(\bar{x})=E \frac{x_{1}+x_{2}+\ldots+x_{n}}{n}=\frac{n E(x)}{n}=E(x)=\bar{x}
$$

e é, portanto, imparcial.

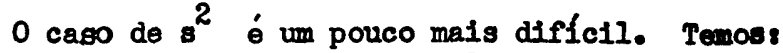

$$
s^{2}=\frac{\sum(x-\bar{x})^{2}}{n}
$$

$=\frac{\sum x^{2}-2 \bar{x} \Sigma x+n \frac{2}{x}}{n}=\frac{\sum x^{2}}{n}-\bar{x}^{2}$.

Consideremos duas variáveis, $x \in I$, não independentes.

Temos 8

$E(\bar{x} . \bar{y})=\mathrm{E}\left[\frac{x_{1}+x_{2}+\ldots+x_{n}}{n} \cdot \frac{y_{1}+y_{2}+\ldots+y_{n}}{n}\right]$

$=\frac{1}{n^{2}}=\left[\left(x_{1}+x_{2}+\ldots+x_{n}\right)\left(y_{1}+y_{2}+\ldots+y_{n}\right)\right]$,

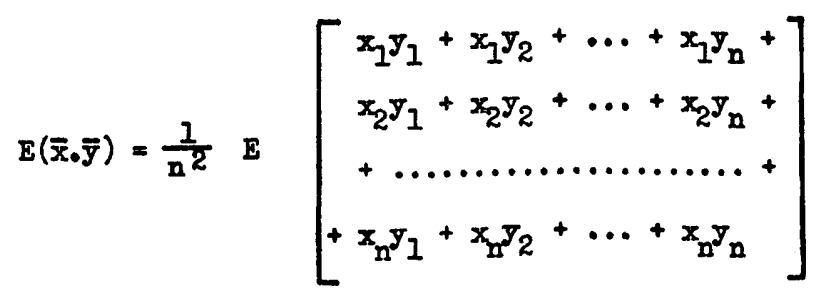


Nos $n$ produtos $x_{1} \bar{y}_{1}, x_{2} y_{2}, \ldots, x_{n} y_{n}$, as variáveis são correlacionadas. Mas nos $\underline{n}^{2}-n$ restantes não pode ha ver correlação. Logo temos

$$
\begin{aligned}
E(\bar{x} \cdot \bar{y}) & =\frac{1}{n^{2}}\left[n E(x y)+\left(n^{2}-n\right) E(x) E(y)\right] \\
& =\frac{E(x y)+(n-1) E(x) E(y)}{n}
\end{aligned}
$$

Para $x=y$ temos, então,

$$
E\left(x^{2}\right)=\frac{E\left(x^{2}\right)+(n-1) x^{2}}{n}
$$

Logo, vamos obter

$$
\begin{aligned}
E\left(s^{2}\right) & =E\left[\frac{\sum x^{2}}{n}-\frac{2}{x}\right] \\
& =\frac{E\left(\sum x^{2}\right)}{n}-E\left(x^{2}\right) \\
& =\frac{n E\left(x^{2}\right)}{n}-\frac{E\left(x^{2}\right)+(n-1) \frac{2}{x}}{n} \\
& =\frac{n-1}{n}\left[E\left(x^{2}\right)-x^{2}\right]=\frac{n-1}{n} \sigma^{2} .
\end{aligned}
$$

Logo $s^{2}$ i uma estimativa tendenclosa de $\sigma^{2}$. Mas

$$
s_{1}^{2}=\frac{\sum(x-\bar{x})^{2}}{n-1}=\frac{n}{n-1} \mathrm{~s}^{2}
$$

é imparcial, pois temos então

$E\left(s_{1}^{2}\right)=\frac{n}{n-1} E\left(s^{2}\right)=\frac{n}{n-1} \cdot \frac{n-1}{n} \sigma^{2}=\sigma^{2}$.

E fica demonstrado elementarmente porque, ao estimar a variância, se divide a soma dos quadrados dos desvios por $n-1$, e não por $n$.

\section{6 - VARIAVEL CASUAL CONTÍNUA}

Quando o conjunto C, campo de definição da variável casual $x$, é do tipo do contínuo, somos obrigados a substituir as 
probabilidades por diferenciais e as somatórias por integrais.

Sendo $y=f(x)$ a equação de uma curva de frequências, a probabilidade de obter um valor entre $x-\frac{1}{2} d x$ e $x+\frac{1}{2} d x$
será

$$
\mathrm{dF}=\mathrm{f}(\mathrm{x}) \mathrm{dx} .
$$

A esperança matemática de uma função $g(x)$ será, por definição,

$$
E[g(x)] \int_{-\infty}^{\infty} g(x) f(x) d x .
$$

No caso da curve normal temos

$$
d P=\frac{1}{\sigma \sqrt{2 \pi}} e^{-\frac{(x-\bar{x})}{2 \sigma^{2}}} d x \text {. }
$$

A média será

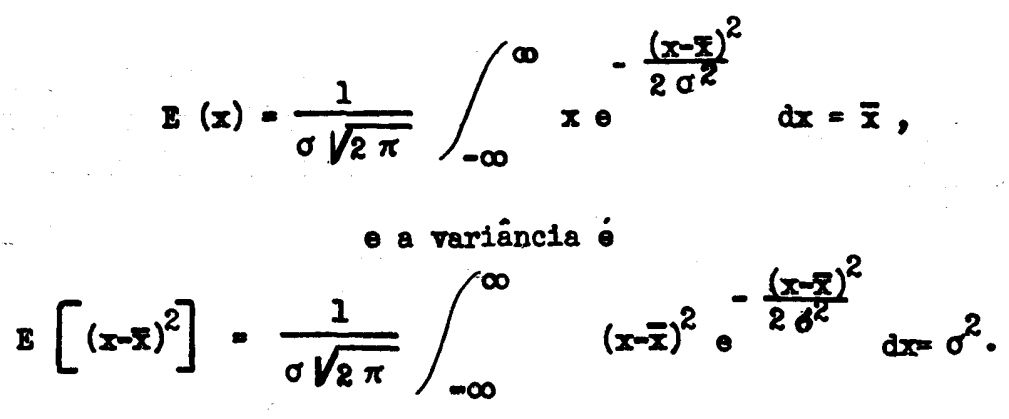

t importante a funcão caraterfistica

$$
\begin{gathered}
\sigma(t)=E\left(e^{1 t x}\right)=\int_{-\infty}^{\infty} e^{i t x} f(x) d x, \\
\text { na qual } 1=\sqrt{-1} .
\end{gathered}
$$

Pode-se demonstrar $(1,18$ vol., pp. $91-94)$ que

$$
f(x)=\frac{1}{2 \pi} \int_{-\infty}^{\infty} \phi(t) \cdot e^{-1 t x} d x .
$$


No caso de $\underline{n}$ variáveis independentes $x_{1}, x_{2}, \ldots, x_{n}$. prova-se fàcilmente (1, 12 vol., pp. 104) que

$$
\begin{gathered}
\phi(t)=\phi_{1}(t) \cdot \phi_{2}(t) \ldots \phi_{n}(t), \\
\phi_{j}(t)=\int_{-\infty}^{\infty} e^{1 t x_{j}} f\left(x_{j}\right) d x_{j}, \\
\text { para } j=1,2, \ldots, n .
\end{gathered}
$$

No caso de uma distribuição normal de média zero e deg v10 "standard" o a função caraterf́stica é

$$
\phi(t)=\frac{1}{\sigma \sqrt{2 \pi}} \int_{-\infty}^{\infty} e^{1 t x} e^{-\frac{x^{2}}{2 \sigma^{2}}} d x=e^{-\frac{1}{2} t^{2} \sigma^{2}}
$$

\section{7 - DISTRIBUIÇÃO DA MÉDIA}

Consideremos a média $\mathrm{x}$ de uma amostra extraída de uma população normalmente distribuída com média zero e desvio "standard" $\sigma$. Qual será a distribuição dessa média $\mathrm{x}$ ?

Temos

$$
\begin{aligned}
z & =\frac{x_{1}+x_{2}+\ldots+x_{n}}{n} \\
& =\frac{x_{1}}{n}+\frac{x_{2}}{n}+\ldots+\frac{x_{n}}{n}
\end{aligned}
$$

Para a primeira variável $x_{1}$ vamos ter

$$
\begin{aligned}
\phi_{1}(t) & =\frac{1}{\sigma \sqrt{2 \pi}} \int_{-\infty}^{\infty} e^{1 t_{n}^{x}} e^{-\frac{x^{2}}{2 \sigma^{2}}} d x \\
& =\frac{1}{\sigma \sqrt{2 \pi}} \int_{-\infty}^{-\left(\frac{x^{2}}{2 \sigma^{2}}-\frac{1 t}{n} x\right)} e^{d x_{0}}
\end{aligned}
$$




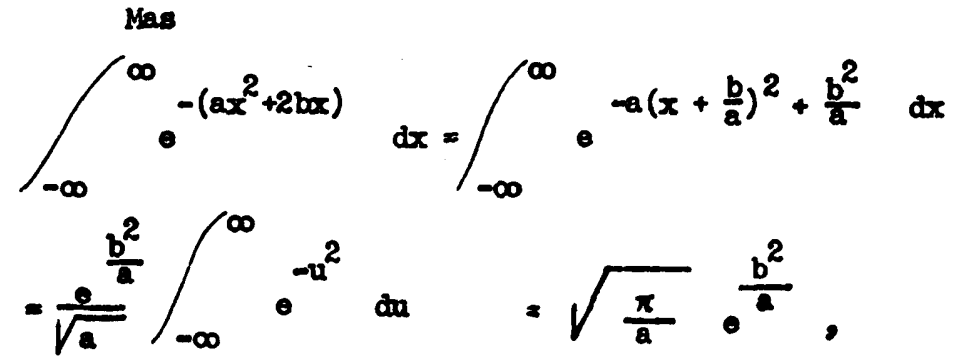

po1s, como se pode demonstrar ( 4, pp. 5-7), a última inte gral vale $\sqrt{\pi}$.

No caso acima temos $a=\frac{1}{2 \sigma^{2}}, b=-\frac{1 t}{2 a} \cdot$ Logo, va

mos ter

$\phi_{1}(t)=\frac{1}{\sigma \sqrt{2 \pi}} \cdot \sqrt{2 \pi \sigma^{2}} 0^{-\frac{t^{2} \sigma^{2}}{2 n^{2}}}=e^{-\frac{t^{2} \sigma^{2}}{2 n^{2}}}$.

Logo
$\phi(t)=\phi_{1}(t) \cdot \phi_{2}(t) \cdots \phi_{n}(t)=\theta^{-\frac{t^{2} \sigma^{2}}{2 n}}$

Por comparação com a função característica da distribuição normal, concluimos logo que a distribuição de $\mathrm{x}$ se dá segundo uma nova curva normal com a mesma média zero e com $\sigma \quad 2$

variância igual a - isto é, a distribuição é dada pela diferencial

n

$$
d F=\frac{\sqrt{n}}{\sigma \sqrt{2 \pi}} e^{-\frac{x^{2} n}{2 \sigma^{2}}} d x .
$$

8. $\triangle$ ESPERANCA DE $\mathrm{s}^{2}$ - Jả sabemos que

$$
\theta^{2}=\frac{\sum(x-\bar{x})^{2}}{n}=\frac{\sum x^{2}}{n}-x^{2} \text {. }
$$




$$
\begin{aligned}
& \text { Logo temos } \\
& E\left(s^{2}\right)=\frac{E\left(\Sigma x^{2}\right)}{n}-E\left(x^{2}\right)=E\left(x^{2}\right)-E\left(x^{2}\right) \\
& E\left(x^{2}\right)=\frac{1}{\sigma \sqrt{2 \pi}} \int_{-\infty}^{\infty} \cdot x^{2} e^{-\frac{x^{2}}{2 \sigma^{2}}} d x \\
& =\frac{0}{\sigma \sqrt{2 \pi}} \int_{-\infty}^{\infty} x^{2} e^{-\frac{x^{2}}{2 \sigma^{2}}} d x \text {. } \\
& E\left(x^{2}\right)=\frac{2 \sigma^{2}}{\sqrt{\pi}} \int_{-\infty}^{\infty} u^{\frac{1}{2}} e^{-u} d u=\frac{2 \sigma^{2}}{\sqrt{\pi}} \Gamma\left(\frac{3}{2}\right)=\sigma^{2} \text {. }
\end{aligned}
$$

Do mesmo modo provamos que

$$
\mathrm{E}\left(\bar{x}^{2}\right)=\frac{\sqrt{n}}{\sigma \sqrt{2 \pi}} \int_{-\infty}^{\infty} x^{2} e^{-\frac{x^{2} n}{2 \sigma^{2}}} d x=\frac{\sigma^{2}}{n} \text {. }
$$

\section{Logo temos}

$$
\mathrm{E}\left(\mathrm{s}^{2}\right)=\sigma^{2}-\frac{\sigma^{2}}{n}=\frac{n-1}{n} \sigma^{2},
$$

de qude se segue que uma estimativa imparcial da variância sera

$$
\begin{aligned}
& s_{1}^{2}=\frac{n}{n-1} s^{2}=\frac{\sum(x-\bar{x})^{2}}{n-1} . \\
& 9 \text { - UM CASO MAIS GERAL }
\end{aligned}
$$

Vamos supor que a amostra de $n$ elementos encerra $m$ amostras parciais, cada uma com $k$ elementos. E' claro que temos

$$
\mathrm{n}=\mathrm{m} . \mathrm{k} \text {. }
$$

Em cada amostra parcial poderemos obter uma estimativa da variância. 


$$
s_{j}^{2}=\frac{\sum_{i=1}^{k}\left(x_{1 j}-x_{j}\right)^{2}}{n-1}
$$

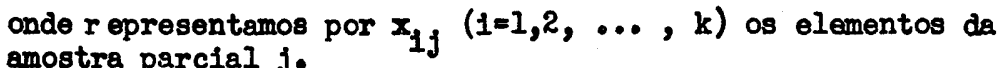

Vamos admitir a hipótese de que

$$
E\left(s_{j}^{2}\right)=\sigma^{2} \text {, }
$$

para $j=1,2, \ldots, m$, isto $\dot{e}$, que tódas as amostras parciais nos dão estimativas de uma mesma variáncia $\sigma^{2}$. Vamos mos trar que

$$
s^{2}=\frac{\Sigma s_{i}^{2}}{m}
$$

é uma estimativa imparcial de $\sigma^{2}$. Com efeito teremos

$$
E\left(s^{2}\right)=E\left[\frac{\Sigma_{1}^{2}}{m}\right]=\frac{m \sigma^{2}}{m}=\sigma^{2}
$$

Kas então teremos ainda

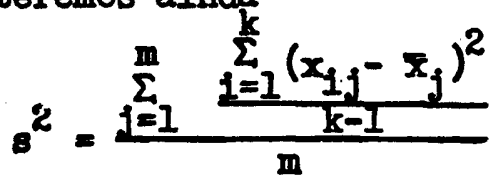

$$
\begin{aligned}
& \frac{\sum_{j=1}^{m} \sum_{i=1}^{k}\left(x_{i j}-\bar{x}_{j}\right)^{2}}{m k-m} \\
= & \frac{\sum_{j=1}^{m} \sum_{i=1}^{k}\left(x_{i j}-\bar{x}_{j}\right)^{2}}{n-m}
\end{aligned}
$$

Logo, quando se consideram $n$ elementos distribuídos em $m$ amostras parciais e se calculam desvios em relação às suas médias, a estimativa da variância será dada pela soma dos quadrados dos desvios dividida por $n-m$, que é o grau de liberdade da estimativa. 


\section{0 - BIBLIOGRAFIA CITADA}

1-KENDALL, Maurice G. - The advanced Theory of Statistics. 3a. edição, 1947. Londres.

2 - USPENSKY, J. V. - Introduction to Mathematical Probability. 1a. edição, 1937. Nova York.

3-CASTELnUOVO, Guido - Calcolo delle Probabilitá. 3a. edição, 1947. Bolonha.

4-PIMENTEL GOMES, Frederico - Introdução ao Estudo dos Derigrais. 1948. Piracicaba. 